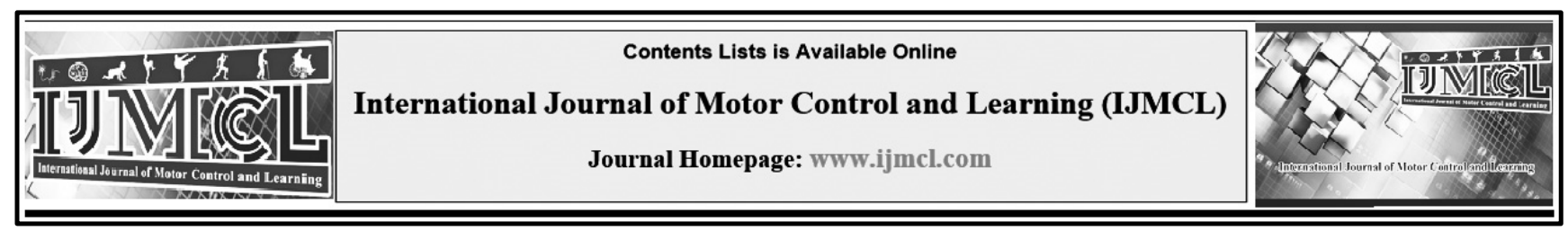

\title{
Psychological Characteristics of Iranian Para-athletes
}

\author{
Mohammad VaezMousavi ${ }^{\mathrm{a}}$, Afrooz Mousavi ${ }^{\mathrm{i} *}$, Fariba Mohammadi ${ }^{\mathrm{c}}$
}

${ }^{a}$ Prof. of Sport Psychology, Physical Education and Sport Sciences Department, Imam Hossein University, Tehran, Iran.

${ }^{\mathrm{b}} \mathrm{Ph} . \mathrm{D}$. in Sport Psychology, National Olympic Academy, Tehran, Iran.

${ }^{\mathrm{c}}$ Assistant Prof. of Sport Injuries and Corrective Exercises, Sports Medicine Research Centre, Sport Sciences Research Institute, Tehran, Iran.

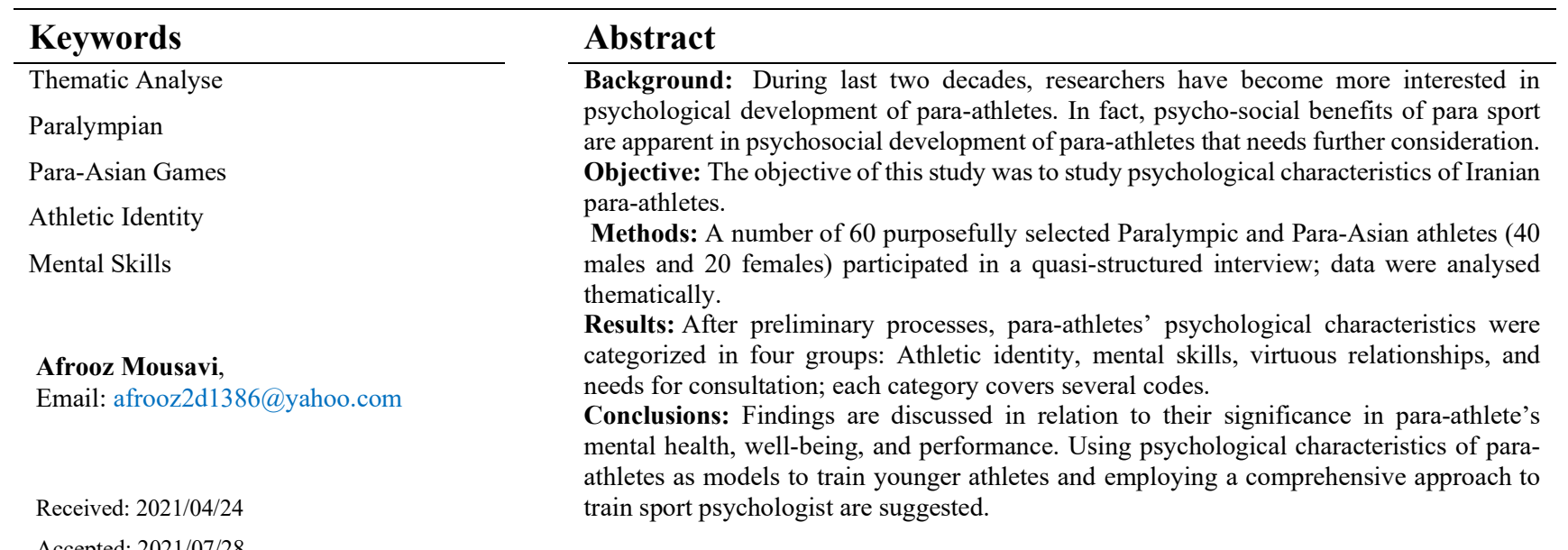

\section{Introduction}

Sport provides opportunities to explore abilities, to experience success, to develop social communications, to satisfy the needs for belonging and acceptance, and to experience joy; which all are valuable for para-athletes (Cheon, Reeve, Lee, \& Lee, 2015). Through improving self-confidence in inter-individual relationships and improving functional skills, sport may develop self-efficacy, which in turn expands capabilities to manage life (Ashton-Shaeffer, Gibson, Autry \& Hanson, 2001) and improve self-esteem (Greenwood, Dzewaltowski, \& French, 1990).
As athletes with disabilities join professional sport, they form a new identity (Peers, 2012) which affects their self-esteem (Van de Vliet, Biesen, \& Vanlandewijck, 2008). Being an elite athlete, develops a more comprehensive identity that potentially can improve individual abilities (Huang \& Brittain, 2006). According to Hatzler (1990), Wheelchair athletes achieve functional independence through improved self-confidence, improved self-esteem, and improved perceived social acceptance. In fact, interacting with teammates in para sport experiences, advances 
characteristics which eventually improves the sense of belonging to the society (Berger, 2008).

Sport participation is a way to adapt with disability (Kirkby, 1995). In fact, psycho-social benefits of para sport are of great importance, in terms of social acceptance, life satisfaction, and recognition of their physical limitations (De Cruz, Spray, and Smith, 2017). According to Van de Vliet (2012), the importance of psychological aspects of sport for athletes with disability are the same as its medical aspects.

In last two decades, researchers became more interested in psychological development of paraathletes. Based on the Holistic athletic career model (Wylleman, Reints, \& De Knop, 2013), which gives the same credit for athletes' well-being as to their performance, participation in para sport helps athletes to improve their quality of life and psychological welfare; however, in sport stressful situations, para-athletes need skills to protect their performance and safeguard their mental health. Professional sport experience, gained prior to incident of disability, could help skill acquisition and provide conditions in which new para sport requirements are well adapted (Dehghansai, Lemez, Wattie, and Baker, 2017). Psychological factors like mental toughness and self-esteem, social factors like coaches' behaviors, and environmental factors like practice conditions could affect sport performance and psychological health (Martin, Malone, and Hilyer, 2011; Martin, 2008; Martin, 2002). Para athletes are constantly coping with bodily pains, performance limitations, psychological stressors, health risks, sport injuries, and unfair expectations (fagher, Jacobsson,
Timpka, Dahlstrom, and Lexell, 2016). According to Powell and Myers (2017) practicing in protective environment, helps para-athletes improve their mental toughness which they may need in demanding situations. It seems that overcoming traumatic events, may help para-athletes to gain such an insight which enables them meeting challenging situation, even better than able body athletes (Martin, Malone, and Hillyer, 2011). Therefore, psychological advantages acquired in para sport are used by para-athletes to cope with every sort of stressors. Ascertaining these characteristics in para-athletes is of value.

Researchers have already studied coping styles (Campbell and Jones, 2002); motivation (Wu and Williams, 2001); personality and mood (Martin, Malone, and Hillyer, 2011); sport identity (Huang \& Brittain, 2006); post-traumatic growth (Crawford, Gayman, Tracey, 2014); and mental skills (Martin and Malone, 2013) in para-athletes. These studies helped to appreciate some aspects of athletic qualities, but the comprehensive psychological profile of para-athletes which includes psychological needs is yet to be studied.

As the level of practice or competition increases, the risk of negative incidents similar to injury, overtraining, burnout, anxiety, and eating disorders also increases, which affects paraathletes' health and well-being (Kenta and Corban, 2014). Most athletes need to control their chronic pain, prevent sport injuries, and cope with sleep disturbances (Martin, 2017). Para-athletes face challenges in air travelling, living in the camps or game villages, and city transportations. Wheelchair para-athletes may develop pressure sores because 
of extended sitting. Para-athletes with spinal injury experience problems in adjusting body temperature (MacCann, 1996) therefore in travelling to a place with different climates they may face psychological stress (Campbell and Jones, 2002).

Sometimes, gender, would be also a challenge for para-athletes. Comparing male to female paraathletes, it was found that females face more cultural challenges than males; in some cultures, female sport is not of significance and is not considered as part of feminine values and competences (Shakespeare, Gillespie-Sells, Davies, 1996).

Psychological studies, emphasizing paraathletes, do not have a long history (Kenta and Corban, 2014). Most of these studies examines the advantages of sport activities for people with disability (Williams, Smith, \& Papathomas, 2014; Rimmer, \& Marques, 2012; Ginis, Jetha, Mack, \& Hetz, 2010; Ashton-Shaeffer, et. al, 2001; Caddick, \& Smith, 2014); however, to improve paraathletes' well-being and mental health, it will be necessary to study both psychological excellences which para-athletes may develop by participating in para sports, and also psychological characteristics which para-athletes need to develop to be able to cope with para sports' challenging situations.

Iranian delegation has participated in eight Paralympics and in last one, Rio, 2016, ranked 15th, earning 24 gold medals. In peoples' view point, para sport has a high cultural and social value; therefore, social, educational, political, and economical departments in the country pay a distinctive attention to para sports. In some para sports, Iranian athletes rank first in world competitions and in Paralympic; therefore, studying psychological characteristics of paraathletes that most of them are prosperous Paralympians, not only could serve as a model for young athletes, but also may be used by researchers for further studies. Investigating psychological features of para-athletes with the purpose of assessing their psychological superiorities and psychological needs, the present study is in a quest of answering to this question that what the most apparent psychological features of Iranian paraathletes are.

\section{Method}

\section{Participants}

A number of 60 para-athletes (40 males and 20 females) took part in the study, including 10 from para-archery ( 3 males and 7 females), 11 from para-shooting ( 3 males and 8 females), 3 from paratennis ( 3 males), 5 from para-taekwondo ( 5 males), 5 from para-swimming (5 males), 9 from 5-aside football (9 males), 9 from para-judo ( 9 males), and 8 from Goal-ball ( 3 male and 5 females). The age of the participants ranged from 18 to 49 years old; the education level ranged from high school diploma to master of art/science; the type of disability comprised of amputee, limb loss, limb deficiency, muscle weakness (spina bifida, spinal cord injury, and muscle dystrophy), visual impairments (partial sighted or completely blind); and the marital status of the participants included single, engaged, married, and divorced.

Including criteria comprised all following three conditions: 1) being a member of a national team; 
2) participation in Rio Paralympic games (2016), or Incheon Para-Asian games (2014), or London Paralympic games (2012), or Guangzhou ParaAsian games (2010); and 3) participation in Jakarta
Para-Asian games (2018) training camps. Demographic characteristics of participants are summarized in Table 1.

Table 1. Characteristics of Para-Athlete Participants.

\begin{tabular}{|c|c|c|}
\hline & $\begin{array}{r}\text { Athlete Demographics } \\
\end{array}$ & Para athletes \\
\hline \multirow{2}{*}{ Gender } & Men & 40 \\
\hline & Women & 20 \\
\hline \multirow{4}{*}{ Age } & $18-24$ & 20 \\
\hline & $25-34$ & 27 \\
\hline & $35-44$ & 12 \\
\hline & $45-49$ & 1 \\
\hline \multirow{3}{*}{ Educational status } & Diploma & 31 \\
\hline & Bachelor & 24 \\
\hline & Master & 5 \\
\hline \multirow{4}{*}{ Marital status } & Single & 24 \\
\hline & Married & 27 \\
\hline & Engaged & 5 \\
\hline & Divorced & 4 \\
\hline \multirow{2}{*}{ Sport } & Individual sport & 36 \\
\hline & Team sport & 24 \\
\hline \multirow{2}{*}{ Highest sporting level } & Paralympic level & 35 \\
\hline & Para Asian Level & 25 \\
\hline \multirow{2}{*}{ Nature of impairment } & Acquired & 45 \\
\hline & Congenital & 15 \\
\hline \multirow{4}{*}{ Type of impairment } & Limb loss or deficiency (e.g., amputee) & 15 \\
\hline & $\begin{array}{l}\text { Muscle weakness (e.g., spinal cord injury, spina bifida, } \\
\text { muscular dystrophies) }\end{array}$ & 16 \\
\hline & Hypertonia, or dystonia (e.g., cerebral palsy) & 3 \\
\hline & Sensory impairment (e.g., blind or visually impaired) & 26 \\
\hline
\end{tabular}

\section{Instruments}

A quasi-structured interview method was used in this study. Stage 1 from the processes of collecting information included questions on family history and the history of participation in professional sport; containing questions aimed to make rapport with the athlete. Stage 2, included indepth interview, pursuing athletes' attitudes towards themselves and their sport experiences. Samples of these questions were: "How do you describe yourself "?; "What are your positive personal assets which help you achieving your goals"?; "In what situations you perform better"?; "What mental skills you used in events you succeed"?; "How do you describe your mental skills"?; "How do you describe your experiences"?; "What superiorities you need to reach to the top"?; "How is your relationship with others working in sport environment"?; "How are your family relationships"?; "What are the benefits of success in sport for you"?. In most cases, the request of "Would you elaborate on this"? was used for in-depth exploration of the concept.

\section{Procedure}

This was a qualitative study using purposeful sampling and phenomenological analysis. To collect data, first, the agreement of National 
Paralympic Committee and the agreement of the head-coaches were obtained. Second, the aim of the study, the benefit of participating in the study, the confidentiality of the data, and the whole procedure were fully described to the athletes and they were asked to cooperate with the procedure and fill in the consent form. Finally, the time of the interview were settled. The duration of the interviews varied from 45 to 55 minutes; based on athletes' conditions and their will, a short rest was given in the midst of it. Interviews continued to the point of theoretical saturation and the contents of the interviews were carefully recorded. Participants were assured of confidentiality of the information and all of them signed the consent form.

\section{Analysis}

Thematic analysis was used to analyze data. Thematic analysis is indeed the analysis of people's emphasis on their feelings, their perceptions, and their experiences, and has many ways of presentation in phenomenological approach. Thematic analysis in this study consisted of six steps which in turn were: familiarization with the data, coding, searching for themes, reviewing themes, defining and naming themes, and inscription of the results (Clarke and Braun, 2013). To improve the credibility of the data, they were returned to the participants after analysis, to verify or to adjust them. To improve the confirmability of the findings, the procedure of the active bracketing was employed to prevent researchers' assumptions from entering to the analysis. To improve the dependability of the findings, two colleagues who were well familiar with the procedure were asked to monitor the encoding, conceptualizing, and defining themes (Creswell, 2013). Finally, participation of both genders with various level of socioeconomic status, in principle improved the transferability of the findings.

\section{Results}

After studying participants' viewpoints, a number of 280 preliminary codes were obtained. Following consolidation of analogous ones, 23 codes were confirmed which were characterized in four categories as "athletic identity", "mental skills", "virtuous relationships" and "needs for consultations". Words in parentheses are participants' statements:

\section{Athletic Identity}

Athletic identity is a psycho-social construct and includes thoughts, beliefs, and communication styles (Erikson, 1968). In this study, based on Brewer, Van Raalte, and Linder (1993), any emphasis on commitment to the sport and to the athletic way of life were encoded as the concept of athletic identity. This category consists of five codes: passion (considering sport as the most important thing in life, loving sport more than anything else, having big dreams about personal advancement in sport, keeping positive attitude toward progression, and so on), determination (training in camps away from loved ones, training even when injured, coping with pain and stress, and so on), self-esteem (cherishing their athletic assets), maturity (learning not to consider physical disability as a limitation, expressing gratitude to other and so on), and mental toughness 
(withstanding stresses accompanied with training/competitions for the sake of beloved goals).

\section{Mental skills}

Although engaging in different sports, mental skills of para-athletes were highly comparable. Mental skills consist of seven codes as follows: proper goal setting (setting realistic and motivational goals), good self-confidence (believing in athletic abilities), skill awareness (awareness about strong points and weak points in their skill), good concentration (not to get distracted when performing and so on), coping with stress (keeping acceptable performing under stress), imagery ability (picturing in mind whatever necessary), and positive self-talk (orientating their perception using positive words).

\section{Virtuous Relationships}

Successful and efficient relationships are significant features of para-athletes. This category consists of good professional relationships (with coaches and other technical staffs, with teammates, and with athletes from other countries), good family relationships (spouse, parents, and so on), and good relationship with God (trusting in God and constantly asking him to assist them).

\section{Need for consultation}

Regardless of all positive features mentioned earlier, para-athletes also need to receive psychological consultations or clinical treatments on several psychological issues. Based on the results of the interview, this category comprised of eight codes as follows: communication problems (problem in communicating with the coach or teammates), family problems (divorce, not having right skills to get married), performance stress (somatic anxiety and cognitive anxiety), concentration vulnerability, sleep disturbance (lack of ability go to sleep the night before competition or when in camp), depression (usually after loss, injury, or a relative's death), adult attention deficit hyperactivity disorder (ADHD), and posttraumatic stress disorder (PTSD) (usually in paraathletes whom their cause of disability was accidents). These four categories are summarized in Table 2.

Table 2. Prominent psychological features of Iranian para-athletes.

\begin{tabular}{ll}
\hline Category & Codes \\
\hline Athletic Identity & $\begin{array}{l}\text { passion, determination, self-esteem, maturity, and } \\
\text { mental toughness }\end{array}$ \\
\hline Mental skills & $\begin{array}{l}\text { proper goal setting, good self-confidence, skill } \\
\text { awareness, good concentration, managing stress, } \\
\text { imagery ability, positive self-talk }\end{array}$ \\
\hline Virtuous Relationships & relationship with coach, with family, and with God \\
\hline Needs for consultation & $\begin{array}{l}\text { Communication problems, family complications, } \\
\text { performance stress, concentration vulnerability, } \\
\text { sleep disturbance, depression, ADHD, PTSD. }\end{array}$ \\
\hline
\end{tabular}




\section{Discussion and Conclusion}

Physical disability in the long run, provides athletes opportunities to improve their mental toughness and make a foundation for those traits which can improve their well-being and performance (Powell and Myers, 2017). In fact, the path to excellence for para-athletes is so special that develops features in their personality which is not possible for able-body athletes. Cox and Davis (1992), comparing psychological skills between wheelchair athletes and able-body athletes, indicated wheelchair athletes scored higher in selfconfidence, motivation, and anxiety control. They also showed that personality characteristics similar to hopefulness, maturity, mental toughness, perseverance, and patience are determinants to accepting disability, coping with adversity, and athletic performance (Cox and Davis, 1992). It is interesting to note that para-athletes who compete at a higher level, have a higher level of personal growth, are more optimistic, and have a broader social support network. These para-athletes are more likely to have a higher level of psychological well-being (MacDougal, Halloran, Sherry, and shields, 2015).

In this study, it was found that regardless of gender and type of disability, para-athletes share characteristics resembling athletic identity, mental skills, virtuous relationships, and also several needs for consultation. Some of these characteristics, were previously mentioned by Powell and Myers (2017), Martin, Malone, and Hillyer (2011), Martin (2012), Martin and Malone (2013), and Jefferies, Gallagher and Dunn (2012). Research indicates that mental skills, self-awareness, and some personality traits directly or indirectly affect athletes' well-being and performance (Martin, 2012). In the present study, personality factors like passion, determination, , self-esteem, maturity, and mental toughness were found as para-athletes' characteristics which are partly in line with what Martin (2012) and Powell and Meyers (2017) previously found.

The importance of athletic identity was previously mentioned by Huang and Brittain (2006) and Crawford, Gayman and Tracey. (2014). Activities in sport programs provides opportunities for significant experiences of independence and self-determination which help to form an athletic identity. According to Crawford, Gayman, and Tracey (2014), sport experiences provide personal growth, self-confidence, social relationships, and athletic identity. In fact, athletic identity improves commitment to sport and affect athletes' thoughts, beliefs, and the way they socialize with others. The history of championship, setting records, and winning medals are determinants of athletic identity; therefore, athletic identity may be the most valuable part of athletes' self. Glorious environment of para games carries positive image about athletes' social statues and its meaningfulness. Athletic life style, a part of athletic identity, was also emphasized in the present study. It contains serving as a role model in the family and neighborhood, training even in problematic situations similar to the time of injury, competing in difficult conditions similar to the time that person losses a member of the family, and accepting injury as a part of the career. Jama (2015) indicated that even injured athletes would not stop 
the athletic rhythm of their life because of the injury. Psychological features of the athletes play roles in developing athletic identity in a synergic manner.

Based on Achievement Goal Theory (Nicholls, 1984), athletes feel competent through mastery in sport performance. According to the results of the present study, para-athletes show mastery in several aspects of mental skills like, selfawareness, good concentration, goal setting, imagery, and positive self-talk. Through these skills, para-athletes are able to manage competitive anxiety. In this study, mental imagery is the skill that is used habitually even by visually impaired athletes. This is in line with Eddy and Mellalieu (2003) who found even class B1 athletes who are totally blind use this technique to improve their performance.

One other finding of this study is that paraathletes are aware of their technical supremacy. A para-archer who won two Paralympic gold medals indicated that constant force control and perfect release are her superiorities, comparing to others. Awareness of technical advantages also means awareness of technical disadvantages, both of them help athletes to come about a proper competition plan. Authors believe that consciousness about what the athlete is able to do in various situations help them to generate some level of creativity necessary for a successful competition (Mousavi and VaezMousavi, 2014).

Successful relationships in the family and in sport environment are other features found in this study. Para-athletes are supported by their family through emotional support, appreciating athletic life style, and holding rational expectations. Most athletes in the present study displayed happiness about the support of their parents or spouses and mentioned this support as a significant factor for their success. Proper relationships in sport is also a matter of consideration. The friendly climate in training camps makes foundation for effective communication. Based on Self-Determination Theory (Ryan \& Deci, 2000), the intrinsic motivation will be adequate, when athletes feel independent, in control, and competent; and also, when they feel successful in relatedness to others (Banack, Sabiston, and Bloom, 2011). According to Garci and Mandich (2005) wheelchair basketball players usually consider teammates, coach, and trainers as their second family. Similar feelings were found in this study to be a foundation for better group interactions which in turn harvests a better group dynamic. One other characteristics of the Iranian para-athletes was not mentioned before in previous studies is belief in God. Deep beliefs are considered as a personality characteristic (Schultz and Schulltz, 2013) and para-athletes in this study declare it as a source of hope and energy. This belief was frequently mentioned by the athletes as a source strength, helping them to try harder. Considering the life style of people in Iranian society, investigators do not find it surprising if athletes follow the common religious beliefs of the general public. Athletes supported by their faiths would be able to give meaning to their endeavors and probably would be able to stay hopeful for a longer period of time. In situations when these athletes have no control over the circumstances, they start praying, hoping the world 
would not stay neutral about their efforts. Since this finding is rather a culture related finding, investigating similar beliefs in other cultures will be of value.

Issues which need consultation are confined in the other class of psychological features of Iranian para-athletes. These issues are communication problems, family complications, performance stress, concentration vulnerability, sleep disturbance, depression, ADHD, and PTSD. Communication problems in the present study include problems in in communicating with teammates in dormitories and problems in communicating with the coach because of perception of double standard or ignorance. Studies indicate that communicating factors could affect para-athlete's well-being and performance (Martin 2002, 2008; Martin, Malone, and Hillyer (2011). Paying attention to this class of problems would help athletes wellbeing and performance.

Comparing to able-body athletes, para-athletes may have different mood states and selfperceptions (Martin, Malone, and Hillyer 2011); therefore, their well-being may be affected by miscommunications or others' ignorance (Lezzoni, 2009). Considering professional environment of competitions, it would be normal if para-athletes experience competitive anxiety, concentration vulnerability, and performance declination. Psychological pressure in training and competition may result in amotivation and depressed mood in which some para-athletes may experience sleep disturbances. Several athletes in the present study reported short periods of depression right before coemption season. ADHD and PTSD were also reported by participants of the present study. These disorders caused them to stop training in periods of time. Paying attention to psychological symptoms similar to confusion or decreased concentration, which indicate more important problem, should be included in training programs of sport psychologists. Since 12 out of 60 para-athletes reported some sort of psychological disorder in the present study, it seems para-athletes' psychological needs cannot be limited to performance enhancement. Therefore, it is suggested that sport psychologist to be prepared for confronting more serious circumstances than teaching mental skills. Using a comprehensive approach in training sport psychologists will enable them to recognize and to intervene in a variety of issues, including clinical issues, and to refer appropriately when is needed.

A limitation of the present study is the fact that the procedure of collecting information was mostly dependent on the athletes' perception of their characteristics, skills, capacities, and needs; the experts' opinion had a minor effect on the results. This may potentially increase some form of bias, inducing by social acceptance phenomenon. The future direction of this study includes using experts' viewpoints by forming focused groups, discussing athletes' notions.

Understanding psychological physiognomies of para-athletes may well illuminate the way for excellence in para sport. Therefore, in training young para-athletes, it is suggested to use the psychological superiorities found in para-athletes as models of achievements. It is also suggested that relationship among gender, age, and the type of disability are studied with the psychological 
conditions of para-athletes. This may provide a better understanding of the challenges' paraathletes face and the coping styles they need to choose.

The high level of stress, existing in the competitive environment of para-sport, could threaten para-athletes' mental health and wellbeing. Therefore, para-athletes' mental needs should not be limited to learning mental skills; rather one should be ready for confrontation psychological disorders as well. This also should be taken in to account in training sport psychologist for working with sport teams.

\section{Conflict of interest}

The authors declare no conflict of interest.

\section{References}

1. Ashton-Shaeffer, C., Gibson, H.J., Autry, C.E. \& Hanson, C.S. (2001). Meaning of sport to adults with physical disabilities: A disability sport camp experience. Sociology of Sport Journal, 18, 95-114.

2. Banack, H.R., Sabiston, C.M. \& Bloom, G.A. (2011). Coach autonomy support, basic need satisfaction, and intrinsic motivation of Paralympic athletes. Research Quarterly for Exercise and Sport, 82(4): 722- 730.

3. Berger, R. J. (2008). Agency, structure, and the transition to disability: A case study with implications for life history research. The Sociological Quarterly, 49(2), 309-333.

4. Brewer, B. W., Van Raalte, J. L., \& Linder, D. E. (1993). Athletic identity: Hercules' ... or Achilles heel? International Journal of Sport Psychology, 24(2), 237-254.

5. Caddick, N., \& Smith, B. (2014). The impact of sport and physical activity on the well-being of combat veterans: A systematic review. Psychology of sport and exercise, 15(1), 9-18.

6. Campbell, E., \& Jones, G. (2002). Sources of stress experienced by elite male wheelchair basketball players. Adapted Physical Activity Quarterly, 19(1), 82-99.

7. Cheon, S. H., Reeve, J., Lee, J., \& Lee, Y. (2015). Giving and receiving autonomy support in a high- stakes sport context: A field-based experiment during the 2012 London Paralympic Games. Psychology of Sport and Exercise, 19, 59-69.

8. Clarke, V. and Braun, V. (2013) Teaching thematic analysis: Overcoming challenges and developing strategies for effective learning. The Psychologist, 26 (2). pp. 120-123.

9. Cox, R.H., \& Davis, R.W. (1992). Psychological skills of elite wheelchair athletes. Palaestra, 8, 1621.

10. Crawford, J.J., Gayman, A.M. \& Tracey, G. (2014). An examination of post-traumatic growth in Canadian and American Para Sport athletes with acquired spinal cord injury. Psychology of Sport and Exercise; 15 (4). 399-406.

11. Creswell, J. W. (2013). Research design: Qualitative, quantitative, and mixed methods approaches. London: Sage publications.

12. De Cruz, N.P., Spray. C.M., Smith, B (2017): Implicit beliefs of disability and elite sport: the para-athlete experience, Qualitative Research in Sport, Exercise and Health, DOI: 10.1080/2159676X.2017.1384753

13. Dehghansai, N., Lemez, s., Wattie, N., Baker, J. (2017). A Systematic Review of Influences on Development of Athletes with Disabilities. Adapted Physical Activity Quarterly, 34, 72 -90.

14. Eddy, K., \& Mellalieu, S.D. (2003). Mental imagery in athletes with visual impairments. Adapted Physical Activity Quarterly. 20(4): 347368.

15. Erikson, E. (1968). Identity Youth and crisis. New York W. W. Norton \& Company.

16. Fagher, K., Jacobsson, J., Timpka, T., Dahlström, Ö., \& Lexell, J. (2016). The Sports-Related Injuries and Illnesses in Paralympic Sport Study (SRIIPSS): a study protocol for a prospective longitudinal study. BMC Sports Science, Medicine and Rehabilitation, 8(1), 28. http://doi.org/10.1186/s13102-016-0053-x.

17. Garci, T.CH, \& Mandich, A. (2005). Going for gold: Understanding occupational engagement in elite-level wheelchair basketball athletes. Journal of Occupational Science; 12(3): 170-175.

18. Gill, D. L., \& Williams, L. (2008). Psychological Dynamics of Sport and Exercise (3rd ed.). Champaign, IL: Human Kinetics.

19. Ginis, K. M., Jetha, A., Mack, D. E., \& Hetz, S. (2010). Physical activity and subjective well-being among people with spinal cord injury: a metaanalysis. Spinal Cord, 48(1), 65.

20. Greenwood, C.M., Dzewaltowski, D.A. \& French, R. (1990). Self-efficacy and psychological wellbeing of wheelchair tennis participants and wheelchair non-tennis participants. Adapted Physical Activity Quarterly, 7, 12-21. 
21. Huang, C. J., \& Brittain, I. (2006). Negotiating identities through disability sport. Sociology of Sport Journal, 23(4), 352-375.

22. Hutzler, Y. (1990). The concept of empowerment in rehabilitative sports. In G. Doll-Tepper, C. Dahms, B. Doll \& H. von Selzam (Eds.), Adapted physical activity: An interdisciplinary approach (pp. 43-51). Berlin: Springer-Verlag.

23. Iezzoni, L. I. (2009). Public health goals for persons with disabilities: looking ahead to 2020. Disability and Health Journal, 2(3), 111-115.

24. Jama, K. M. (2015). A collective case study of athletic identity and successful injury rehabilitation. Master's Thesis in Sport and Exercise Psychology. Department of Sport Sciences University of Jyväskylä. $\quad$ http://urn.fi/URN:NBN:fi:jyu201505011704

25. Jefferies, P., Gallagher, P. \& Dunne, S. (2012). The Paralympic athlete: a systematic review of the psychosocial literature. Prosthetics and Orthotics International, 36(3):278-89. doi: $10.1177 / 0309364612450184$.

26. Kenttä, G., \& Corban, R. (2014). Psychology within the Paralympic Context-Same, Same or Any Different?. Olympic Coach, 25(3), 15-25.

27. Kirkby, R.J. (1995). Wheelchair netball: Motives and attitudes of competitors with and without disabilities. Australian Psychologist, 30, 109-112.

28. Macdougall, H., O'Halloran, P. D., Sherry, E., \& Shields, N. (2015). Needs and strengths of Australian Para-Athletes: Identifying their subjective psychological, social, and physical health and well-being. Sport Psychologist, 30, 112.

29. Martin, J. J. (2002). Training and performance selfefficacy, affect, and performance in wheelchair road racers. Sport Psychologist, 16, 384-395.

30. Martin, J. J. (2008). Multidimensional self-efficacy and affect in wheelchair basketball players. Adapted Physical Activity Quarterly, 25, 1-15.

31. Martin, J. J. (2017). Oxford Research Encyclopedia of Psychology, Edition: 1st, Chapter: Psychological Considerations for Paralympic Athletes, Publisher: Oxford University, Editors: Edmund Acevedo, pp.1-27.

32. Martin, J. J., \& Malone, L. (2013). Elite wheelchair rugby players' mental skills and sport engagement. Journal of Clinical Sport Psychology, 7, 253-263.

33. Martin, J.J., Malone, L.A., \& Hillyer, J.C. (2011). Personality and mood in women's Paralympic basketball champions. Journal of Clinical Sport Psychology, 5, 197-210.

34. Martin, J. (2012). Mental Preparation for the 2014 Winter Paralympic Games. Clinical. Journal of Sport Medicine, 22, 70-73.
35. McCann, B.C. (1996). Thermoregulation in spinal cord injury: The challenge of the Atlanta Paralympics. Spinal Cord, 34, 433-436.

36. Mousavi, A., VaezMousavi, M. (2015). Introducing the Sport Success Scale (SSS). Motor Behavior. 7(19); 123-142. (In Persian)

37. Nicholls, J. G. (1984). Achievement Motivation: Conceptions of Ability, Subjective Experience, Task Choice, and Performance. Psychological Review, 91, 328-346.

38. Peers, D. (2012). Interrogating disability: The (de)construction of a recovering Paralympian. Qualitative Research in Sport and Exercise, 4(2), 175-188.

39. Powell, A. J., \& Myers, T. D. (2017). Developing Mental Toughness: Lessons from Paralympians. Frontiers in Psychology, 8, 1270. http://doi.org/10.3389/fpsyg.2017.01270

40. Rimmer, J. H., \& Marques, A. C. (2012). Physical activity for people with disabilities. The Lancet, 380(9838), 193-195.

41. Ryan, R. M. \& Deci, E. L. (2000). Selfdetermination theory and the facilitation of intrinsic motivation, social development, and wellbeing. American Psychologist, 55, 68-78.

42. Schultz, Duane, P. and Schultz, Sydney, E. (2013). Theories of Personality. Wadsworth.USA.

43. Shakespeare, T., Gillespie-Sells, K. and Davies, D. (1996). The sexual politics of disability: Untold desires London: Cassell.

44. Van de Vliet, P. (2012). Paralympic athlete's health. British Journal of Sports Medicine, 46, 458459.

45. Van de Vliet, P., Biesen, D. V., \& Vanlandewijck, Y. C. (2008). Athletic identity and self-esteem in Flemish athletes with a disability. European. Journal of Adapted Physical Activity, 1, 9-21.

46. Williams, T. L., Smith, B., \& Papathomas, A. (2014). The barriers, benefits and facilitators of leisure time physical activity among people with spinal cord injury: a meta-synthesis of qualitative findings. Health Psychology Review, 8(4), 404425.

47. Wu, S. K., \& Williams, T. (2001). Factors influencing sport participation among athletes with spinal cord injuries. Medicine \& Science in Sports \& Exercise, 33(2), 177-182.

48. Wylleman, P., Reints, A., \& De Knop, P. (2013). A developmental and holistic perspective on athletic career development. In Managing high performance sport (pp. 191-214). Routledge. 